\title{
The Effect of Variable Viscosity on Unsteady Free Convective Fluid Flow over a Stretching Surface in the Presence of Thermal Radiation and Viscous Dissipation
}

\author{
Nyakebogo Abraham Osogo ${ }^{1}$, Kerongo Joash Morara $^{2}$ \& Obogi Robert Karieko ${ }^{3}$ \\ ${ }^{1,2,3}$ Mathematics and Actuarial Sciences Department \\ Kisii University, 408-40200, Kisii
}

Kenya

\begin{abstract}
The unsteady free convective flow of an incompressible, viscous, electrically conducting fluid over a vertical stretching surface with variable viscosity and thermal radiation in the presence of viscous dissipation is considered. The fluid is assumed to be gray, absorbing emitting but non-scattering medium. Thus, the Rosseland approximation is used to describe the radiative heat flux in the energy equation. The viscosity of the fluid is assumed to be an inverse linear function of temperature. Specifically, the study is aimed at determining the effects of variable viscosity parameter $(\delta)$, Eckert number $\left(E_{C}\right)$ and radiation parameter $(R)$ on temperature distribution profiles, while holding Prandtl number constant that is $\operatorname{Pr}=0.7$ corresponding to air. The specific energy equation governing the fluid flow is solved numerically by finite difference method. The nonlinear partial differential equation is solved numerically by finite difference method with. MATLAB code is used to solve the Forward Time and Centered Space (FTCS) scheme. Further, stability and consistency analysis are performed on the FTCS scheme to determine the convergence of the scheme. Results for temperature distribution profiles are presented graphically for different values of variable viscosity $(\delta)$, Eckert number $\left(E_{C}\right)$, radiation parameter $(R)$. The findings are that an increase in variable viscosity and Eckert number causes an enhancement of temperature distribution profiles whereas an increase in radiation parameter leads to a decrease in temperature distribution profiles. The FTCS scheme was found to be unconditionally stable and consistent. The results obtained in this study follow expected trends and are in good agreement with some previous studies.
\end{abstract}

Key words: Variable Viscosity, Magneto hydrodynamics, Stretching Surface, Thermal Radiation, Von-Neumann Analysis, FTCS Scheme.

\section{INTRODUCTION}

Fluid flow over a stretching surface plays a very important role in the field of metallurgy and chemical engineering and in particular, the extrusion of polymer sheet from a die or in the drawing of plastic films. During the manufacture of these sheets, the melt materials from a slit is stretched continuously to achieve the desired thickness, Alinejad and Samarbakhsh [1]. The properties of the final product depend to a great extent on the rate of cooling. The rate of cooling is fundamentally influenced by the type of fluid adjacent to the boundary layer surface, Jafar et al, [2].

Great interest has been shown in the study of heat transfer over a stretching surface. Pop and Na [3] studied unsteady flow past a stretching sheet. Nazar et al [4] presented a study on unsteady boundary layer flow of an incompressible micropolar fluid with the assumption that the stretching velocity varied linearly with the distance along the sheet. Elbashbeshy and Aldawoody [5] focused on unsteady flow over a stretching surface with variable heat flux while taking into account a heat source or sink. Phakirappa, et al [6] analyzed free convective viscous fluid flow and heat transfer flow past a stretching sheet taking into account heat sink and internal heat generation. A study on partial slip flow and heat transfer over a stretching sheet in a nanofluid was 
carried out by Sharma, et al [7]. Vajravelu, et al [8] studied the unsteady convective boundary layer flow of a viscous fluid on a vertical surface for which numerical solutions were presented.

Thermal radiation is pertinent in the studies involving science and engineering. Major processes in engineering occur at a high temperature especially in designing of energy conversion equipment, Reddy, et al [9]. Malapati and Polarapu [10] analyzed the nonlinear unsteady MHD flow of viscous, incompressible and electrically conducting fluid past a vertical porous channel under the influence of thermal radiation and chemical reaction. Sharma and Gupta [11]) carried out a study on the analytical solution of the two-dimensional MHD flow of viscoelastic Jeffrey fluid through impermeable surface in presence of thermal radiation and viscous dissipation taking into consideration heat generation/absorption. Daniel et al [12]) presented magnetohydrodynamics flow of nanofluid towards nonlinear stretched surface with variable thickness in presence of electric field. The analysis took into account viscous dissipation, Joule heating and chemical reaction. Fenuga et al [13] investigated the effects of radiation and Eckert number on MHD flow with heat transfer rate near a stagnation-point region over a nonlinear vertical stretching sheet.

However, the above literature did not consider variation in viscosity which occurs as a result of change in temperature as the fluid flows along the stretching surface. Owing to its importance, studies have been done by a number of researchers. Shit and Haldar [14] investigated the effect of thermal radiation and temperature dependent viscosity on free convective flow and mass transfer of an electrically conducting fluid over an isothermal stretching sheet. They observed that velocity decreased with increase of the magnetic parameter, Prandtl number, thermal radiation parameter, viscoelastic parameter and species diffusion parameter while it increased with the increase of viscoelastic parameter. A numerical model was developed by Salem [15] to study the effects of temperature dependent viscosity on heat and mass transfer flow of magnetohydrodynamic (MHD) micropolar fluids along a permeable stretching surface embedded in a non-Darcian porous medium in the presence of viscous dissipation and chemical reaction. Their results indicated that in the presence of viscous dissipation enhanced variable viscosity parameter caused a rise in the rate of heat transfer near the surface while decreasing the same significantly away from the surface. Pal and Mondal [16] presented a numerical model to study the effects of temperature dependent viscosity and variable thermal conductivity on mixed convection problem. Two cases of wall heating conditions namely, prescribed surface temperature (PST) and prescribed heat flux (PHF) which arise in polymer industries were considered. Their results indicated that enhanced Prandtl number led to the decline in the skin friction coefficient, local Nusselt number and local Sherwood number whereas a rise in Schmidt number lessened the concentration profile in both PST and PHF cases.

The present paper examines the effect of variable viscosity on the unsteady free convective fluid flow over a vertical stretching sheet in the presence of thermal radiation and viscous dissipation. Fluid viscosity is assumed to vary as an inverse linear function of temperature. Using finite difference approximations, the unsteady energy equation is discretized. The discretized equation is solved numerically by finite difference method. The effects of different parameters on temperature distribution profiles are investigated considering a case in which the Prandtl number is held at a constant value, that is, at $\operatorname{Pr}=0.71$ (corresponding to air). The solutions are then analyzed graphically. Further, the stability and consistency analysis of the FTCS scheme developed from the energy equation partial differential equation was done to ascertain that the PDE was well posed.

\section{MATHEMATICAL FORMULATION}

A two-dimensional unsteady incompressible electrically conducting fluid flow over a vertical stretching surface is considered. The flow is infinite along the $x$-axis and the sheet is non-conducting with injection taking place through the porous walls of the channel with uniform velocity $v_{0}$. The y-axis is perpendicular to the sheet in the upward direction and the z-axis is transverse to the $x y$-plane. The continuous sheet moves in its own plane with velocity $U_{w}(x, t)$ and the temperature $T_{w}(x, t)$ distribution varies both along the sheet and time. The fluid flow being studied is free convectional and takes place along the $x$-axis under the action of transverse magnetic field. The temperature at the sheet is maintained at a prescribed constant $T_{w}$. The flow is generated due to a stretching sheet which is caused by applying two equal opposite forces. It is also assumed that no applied or polarization voltages exist since the plate is insulated. Thus $\boldsymbol{E}=0$. To simplify the problem, we assume that there is no variation of flow quantities in the $z$-direction. It is also assumed that the fluid properties are isotropic and constant except for the fluid viscosity which is assumed to vary as an inverse linear function of temperature $T$ in form Salem [15]:

$\frac{1}{\mu}=\frac{1}{\mu_{\infty}}\left[1+\gamma\left(T-T_{\infty}\right)\right]$, that is, $\frac{1}{\mu}=a\left(T-T_{r}\right)$

Where $a=\frac{\gamma}{\mu_{\infty}}$ and $T_{r}=T_{\infty}-\frac{1}{\gamma}$ are constants and their values depend on the state of the fluid.

Under the above assumptions, the continuity and energy conservation equations governing the flow can be expressed as follows (Sharma and Gupta [13] Shateyi and Motsa [17]): 
$\frac{\partial u}{\partial x}+\frac{\partial v}{\partial y}=0$

$\rho C_{P}\left(\frac{\partial T}{\partial t}+u \frac{\partial T}{\partial x}+v \frac{\partial T}{\partial y}\right)=k \frac{\partial^{2} T}{\partial y^{2}}+\mu\left(\frac{\partial u}{\partial y}\right)^{2}-\frac{\partial q_{r}}{\partial y}$

Where $u$ and $v$ are the velocity components of fluid in $x$ and $y$ directions respectively, $\rho$ is the fluid density, $\mu$ is the dynamic viscosity, $\mathrm{k}$ is the thermal conductivity, $C_{P}$ is the specific heat at constant pressure, $T$ is the temperature of the fluid, $q_{r}$ is the component of radiative heat flux.

The boundary conditions are given by:

$$
\begin{aligned}
& t \leq 0, u=0, \quad v=0, \quad T=T_{\infty} \quad \text { for all } y \\
& t>0 u=u_{0}, v=0, T=T_{W} \quad \text { at } y=0 \\
& u \rightarrow 0, v \rightarrow 0, T=T_{\infty}, \text { as } y \rightarrow \infty
\end{aligned}
$$

The stretching sheet is of infinite length along $\mathrm{X}$-axis, hence $\frac{\partial \boldsymbol{u}}{\partial x}=0$. Therefore equation (2) reduces to:

$$
\frac{\partial v}{\partial y}=0
$$

Which upon integration gives

$v=v_{0}$

where $v_{0}$ is the injection velocity.

By Rosseland approximation, radiative heat flux of an optically thin gray fluid $q_{r}$ is expressed as Dulal and Mondal, [18]

$\frac{\partial q_{r}}{\partial y}=-\frac{4 \sigma^{*} \partial T^{4}}{3 \alpha \partial y}$

Where $\sigma^{*}$ is the Stefan-Boltzmann constant and $\alpha$ is the mean absorption coefficient. It is assumed that the temperature differences within the flow are sufficiently small so that the term $T^{4}$ may be expressed as a linear function of temperature. This is achieved by expanding $T^{4}$ in a Taylor series about $T_{\infty}$ and leaving out the second and higher order terms. Thus obtaining:

$T^{4} \cong 4 T T_{\infty}^{3}-3 T_{\infty}^{4}$

Substituting equation (6) into equation (5) yields:

$\frac{\partial q_{r}}{\partial y}=-\frac{4 \sigma^{*} \partial\left(4 T T_{\infty}^{3}-3 T_{\infty}^{4}\right)}{3 \alpha \partial y}=-\frac{16 \sigma^{*} T_{\infty}^{3}}{3 \alpha} \frac{\partial^{2} T}{\partial y^{2}}$

Thus, upon substituting equation (7) into equation (2) gives:

$\rho C_{P}\left(\frac{\partial T}{\partial t}+u \frac{\partial T}{\partial x}+v \frac{\partial T}{\partial y}\right)=k \frac{\partial^{2} T}{\partial y^{2}}+\mu\left(\frac{\partial u}{\partial y}\right)^{2}+\frac{16 \sigma^{*} T_{\infty}^{3}}{3 \alpha} \frac{\partial^{2} T}{\partial y^{2}}$

Factoring in the variable viscosity parameter into equation (9), and re-arranging yields:

$\left(\frac{\partial T}{\partial t}+u \frac{\partial T}{\partial x}+v \frac{\partial T}{\partial y}\right)=\frac{k}{\rho C_{P}}\left(\frac{\partial^{2} T}{\partial y^{2}}\right)+\frac{1}{a\left(T-T_{r}\right) \rho C_{P}}\left(\frac{\partial u}{\partial y}\right)^{2}+\frac{1}{\rho C_{P}} \frac{16 \sigma^{*} T_{\infty}^{3}}{3 \alpha} \frac{\partial^{2} T}{\partial y^{2}}$

The following non-dimensional variables are defined for the present problem:

$y^{*}=\frac{y U_{0}}{v}, \quad u^{*}=\frac{u}{U_{0}}, \theta=\frac{T-T_{\infty}}{T_{w}-T_{\infty}}, \quad v_{0}^{*}=\frac{v_{0}}{U_{0}}, \quad t^{*}=\frac{t U_{0}^{2}}{v}$

The non-dimensional form of the energy equation gives:

$v_{0} \frac{\partial \theta}{\partial y}+u \frac{\partial \theta}{\partial x}+\frac{\partial \theta}{\partial t}=\frac{1}{\operatorname{Pr}} \frac{\partial^{2} \theta}{\partial y^{2}}+E c \delta\left(\frac{\partial u}{\partial y}\right)^{2}+\frac{R}{\operatorname{Pr}} \frac{\partial^{2} \theta}{\partial y^{2}}$ 
where $\operatorname{Pr}=\frac{k}{v \rho C_{p}}$ the Prandtl number, $E c=\frac{U_{0}^{2}}{v \rho C_{p}\left(T_{w}-T_{\infty}\right)}$ Eckert number, $\delta=\frac{1}{a\left(T-T_{r}\right)}$ is the variable viscosity parameter and $R=\frac{16 \sigma T_{\infty}^{3}}{3 \alpha}$ is the radiation parameter.

\section{SOLUTION OF THE PROBLEM}

The coupled non-linear governing equation (11) is solved numerically using the forward time central space (FTCS) difference method which is stable and convergent.

The finite difference form of the energy equation (11) is given as:

$\frac{\theta_{i, j}^{k+1}-\theta_{i, j}^{k}}{\Delta t}+\frac{\theta_{i+1, j}^{k}-\theta_{i-1, j}^{k}}{2(\Delta x)}+\frac{\theta_{i, j+1}^{k}-\theta_{i, j-1}^{k}}{2(\Delta y)}=\left(\frac{1+R}{P r}\right)\left(\frac{\theta_{i, j+1}^{k}-2 \theta_{i, j}^{k}++\theta_{i, j-1}^{k}}{(\Delta y)^{2}}\right)+E c \delta\left[\frac{\left(u_{i, j+1}^{k}\right)^{2}+2 u_{i, j+1}^{k} u_{i, j-1}^{k}+\left(u_{i, j+1}^{k}\right)^{2}}{4(\Delta y)^{2}}\right]$

A mesh size is fixed at $\Delta x=\Delta y=0.1$ and $\Delta t=0.01$ and re-arranging equation (12) yields:

$0.4 \theta_{i, j}^{k+1}=0.4 \theta_{i, j}^{k}-0.2 \theta_{i+1, j}^{k}+0.2 \theta_{i-1, j}^{k}-0.2 \theta_{i, j+1}^{k}+0.2 \theta_{i, j-1}^{k}+\left(\frac{1+R}{P r}\right)\left(\theta_{i, j+1}^{k}-2 \theta_{i, j}^{k}++\theta_{i, j-1}^{k}\right)+$ $E c \delta\left[\left(u_{i, j+1}^{k}\right)^{2}+2 u_{i, j+1}^{k} u_{i, j-1}^{k}+\left(u_{i, j+1}^{k}\right)^{2}\right]$

Initial and boundary conditions are used to determine the values of temperature distribution. The following values are used:

Initial condition:

$\theta(x, y, 0)=1$

and boundary conditions:

$\theta(0, y, t)=0, \quad \theta(5, y, t)=0, \quad 0 \leq x \leq 5$

$\theta(x, 0, t)=0, \quad \theta(x, 2, t)=0, \quad 0 \leq y \leq 2$

The finite difference equation (13) is then solved using MATLAB software.

\section{RESULTS AND DISCUSSION}

The effect of variable viscosity on unsteady magnetohydrodynamic fluid flow over a stretching surface in the presence of thermal radiation and viscous dissipation has been considered in this study. The parameters encountered are assigned numerical values: radiation parameter $R=1,2,3$, variable viscosity parameter $\delta=0.5,1.0$, and 2.0, Eckert number $E c=10,20,30$. The analysis was done for two different values of Prandtl number as illustrated below:

A program was written and run for various values of temperature for finite difference equation (13) using the different values of $R, \delta$ and $E c$ as indicated above. The analysis of the data distribution for $\operatorname{Pr}=0.71$ corresponding to air was done and the results are represented graphically on figures 1, 2 and 3 below. 


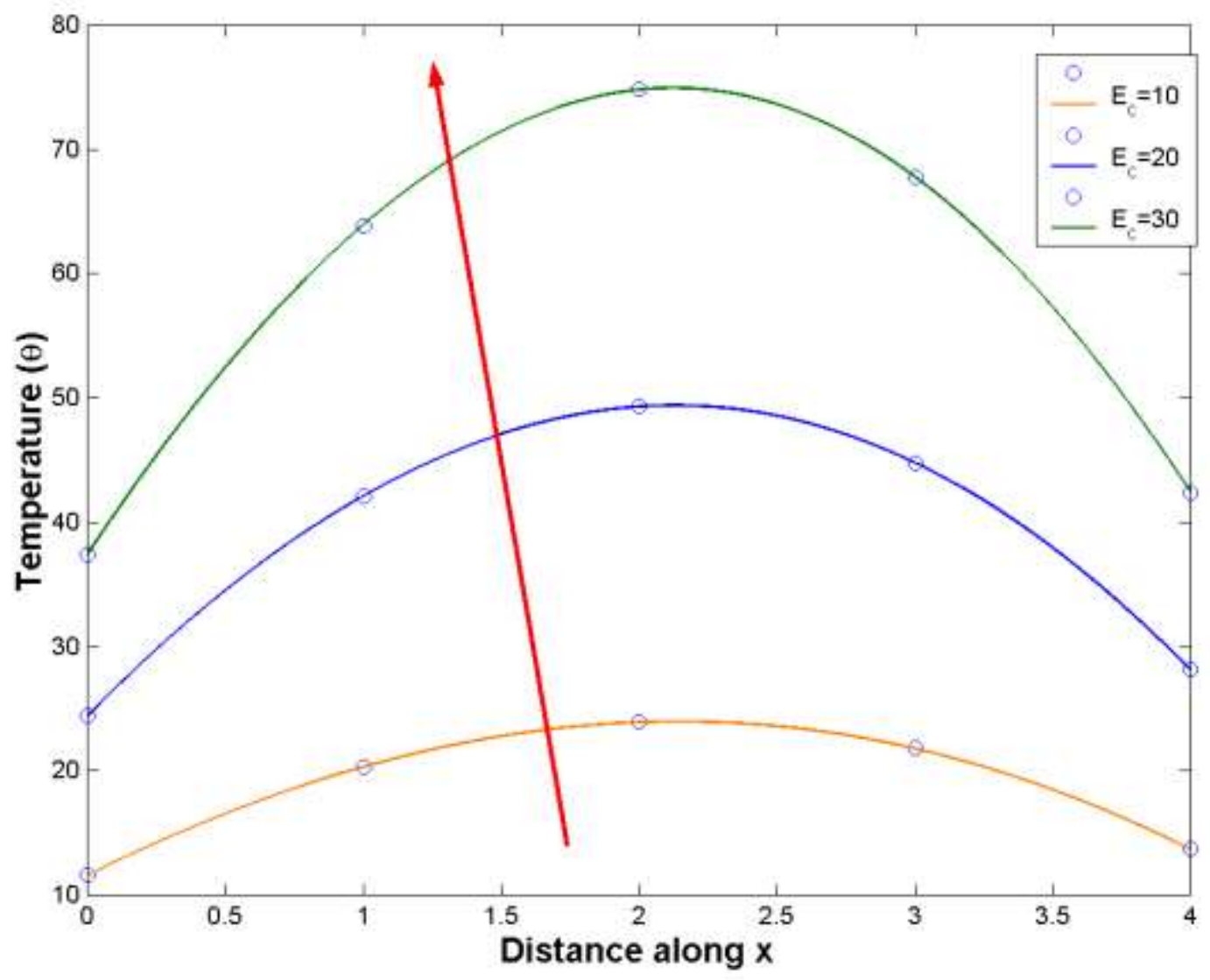

Figure 1: Effects of Eckert number on temperature at $\delta=1, R=1, \operatorname{Pr}=0.71$

The temperature profiles for the Eckert number are displayed in figure 1. From the figure it is clear that as Eckert number increases, the temperature profiles rise. With increase in Eckert number, the fluid temperature near the bottom of the stretched surface increases and then decreases away from the stretched surface. Physically, the Eckert number is a ratio of kinetic energy to the enthalpy. It incorporates the conversion of kinetic energy into internal energy by work done against the viscous fluid stresses. Greater viscous dissipative heat causes a rise in the temperature profile. However, kinetic energy reduces as distance increases in the vertical direction whereas thermal energy difference across the boundary layer increases as distance increase in the same direction. A positive Eckert number implies heating of the fluid as it absorbs heat from the stretching surface leading to thicker thermal boundary layer and cooling of the stretching surface. Thus, the fluid temperature increases as more heat energy is realized from the internal viscous forces. 


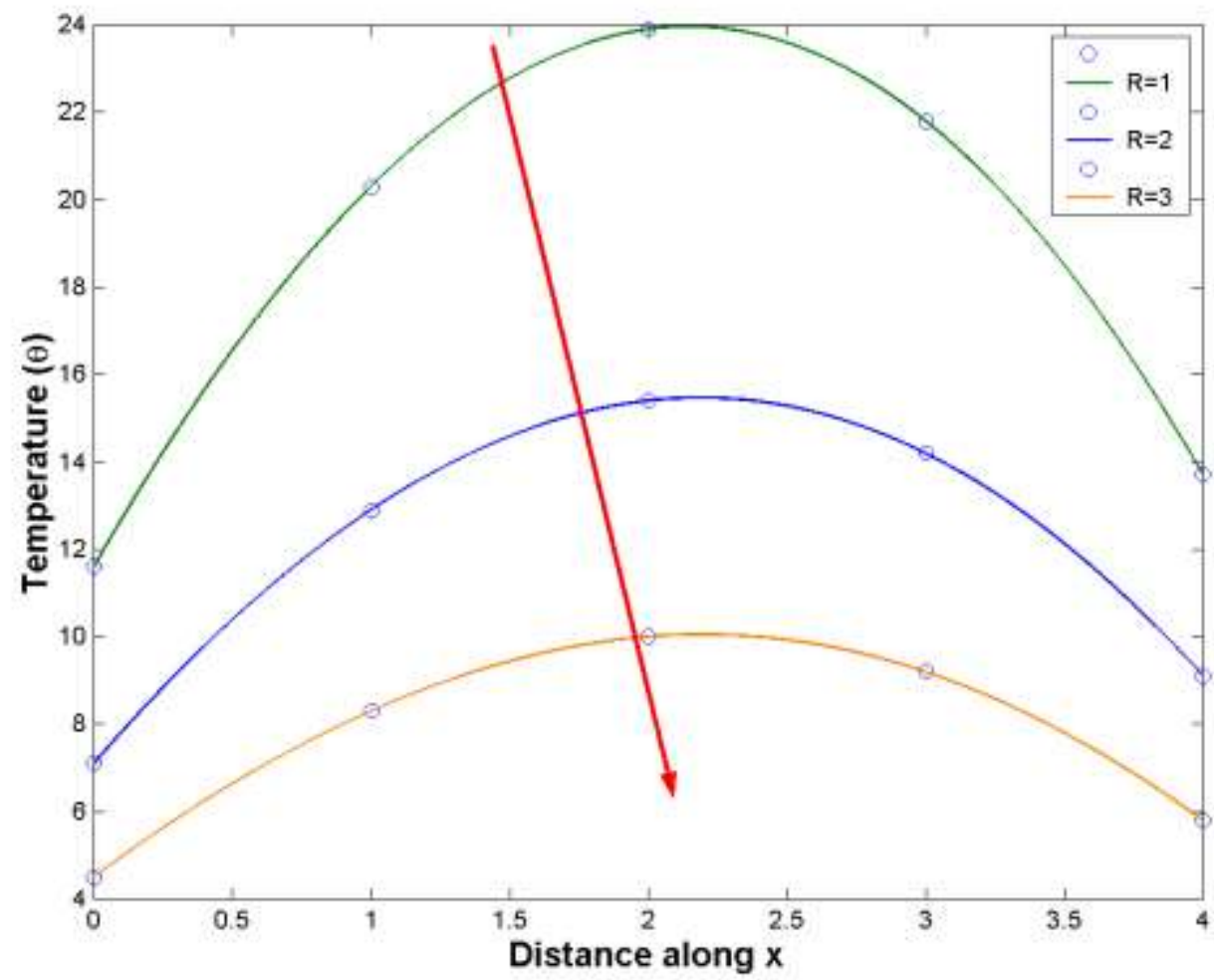

Figure 2: Effects of radiation parameter on temperature at $\delta=1, E c=10, \operatorname{Pr}=0.71$

It is revealed from figure 2 that the temperature decreases as radiation parameter increases. With an increase in radiation parameter, there is a decrease in thermal boundary layer thickness which leads to enhanced rate of heat transfer. This effect can be used to increase the rate of cooling of the sheet when required.

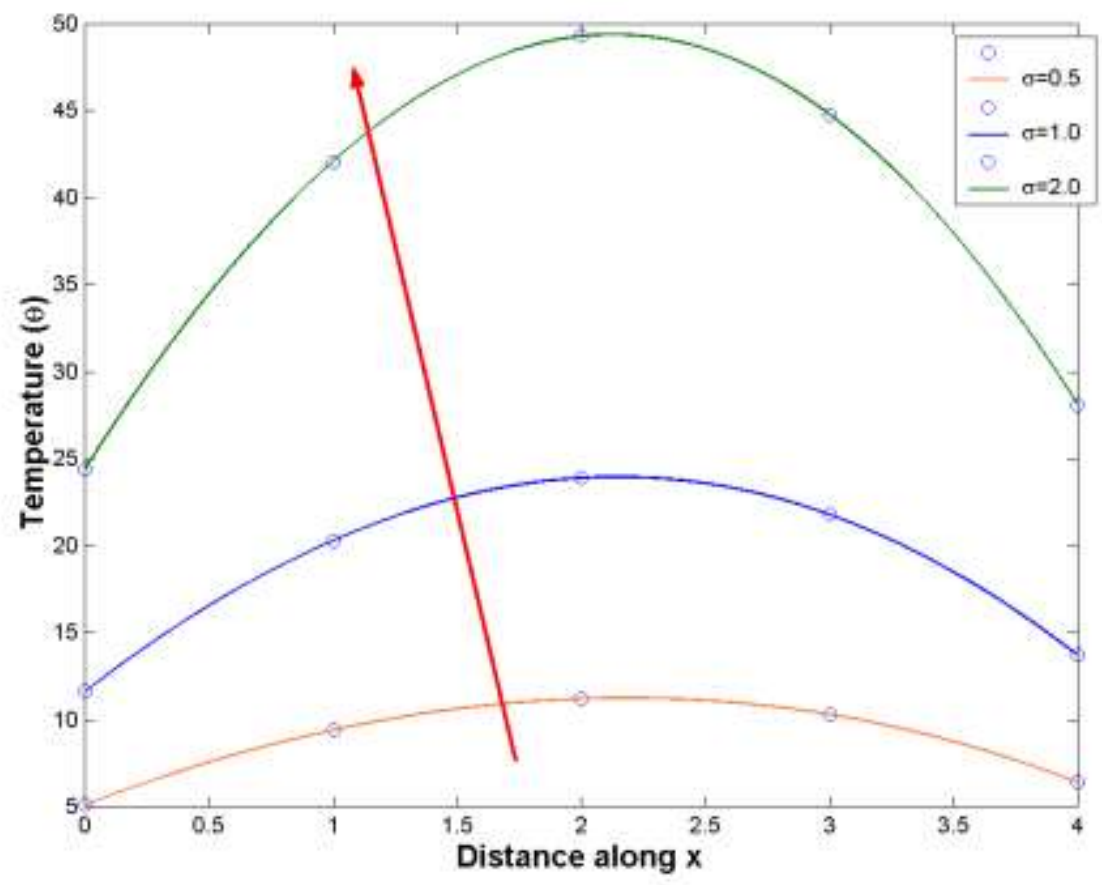

Figure 3: Effects of variable viscosity parameter on temperature at $R=1, E c=10, \operatorname{Pr}=0.71$

Figure 3 illustrates the effect of variable viscosity parameter $\delta$ on dimensionless temperature $\theta$. Increase in variable viscosity parameter implies increased fluid viscosity which in turn leads to an increase in the viscous force which opposes the motion of the 
fluid. This increase in the viscous force leads to an increase in temperature owing to the friction between the surface and the fluid causing generation of more heat. Thus, bringing about increased thickness in the thermal boundary layer.

\section{STABILITY AND CONSISTENCY ANALYSIS}

There are numerous techniques to be utilized for numerical analysis. Finite difference method has been used in this study by developing the FTCS scheme for the energy equation. There is need for determining how well the FTCS scheme performs in terms of stability, consistency and convergence. This study has utilized the Von-Neumann stability analysis and the Taylor's series for the consistency analysis. The details are explained as follows:

\subsection{Von-Neumann Stability Analysis for the Energy Equation FTCS Scheme}

The FTCS numerical scheme for the temperature $\theta(x, y, t)$ is developed for the energy equation (11). Discretization of the equation (11) gives:

$\frac{\theta_{i, j}^{k+1}-\theta_{i, j}^{k}}{\Delta t}+u_{i, j}^{k} \frac{\theta_{i+1, j}^{k}-\theta_{i-1, j}^{k}}{2(\Delta x)}+v_{0} \frac{\theta_{i, j+1}^{k}-\theta_{i, j-1}^{k}}{2(\Delta y)}=\left(\frac{1+R}{P r}\right)\left(\frac{\theta_{i, j+1}^{k}-2 \theta_{i, j}^{k}+\theta_{i, j-1}^{k}}{(\Delta y)^{2}}\right)+E c \delta\left[\frac{\left(u_{i, j+1}^{k}\right)^{2}+2 u_{i, j+1}^{k} u_{i, j-1}^{k}+\left(u_{i, j+1}^{k}\right)^{2}}{4(\Delta y)^{2}}\right]$

Putting $R=\operatorname{Pr}=1, E c \delta \mu=0, \frac{\Delta t}{\Delta x}=\frac{\Delta t}{\Delta y}=\emptyset, \frac{\Delta t}{(\Delta y)^{2}}=\mu$ and multiplying equation (15) by $2 \Delta t$ we obtain:

$2 \theta_{i, j}^{k+1}-2 \theta_{i, j}^{k}+\varnothing \theta_{i+1, j}^{k}-\varnothing \theta_{i-1, j}^{k}+\emptyset \theta_{i, j+1}^{k}-\varnothing \theta_{i, j-1}^{k}=2 \mu \theta_{i, j+1}^{k}-4 \mu \theta_{i, j}^{k}+2 \mu \theta_{i, j-1}^{k}$

Taking the trivial solution as $\theta_{i, j}^{k}=\lambda^{t} e^{I x \Psi} e^{I y \Psi}$ when $x, y>0, I=\sqrt{(-1)}$, Lapidus and Pinder [19] and substituting in equation (17) results to:

$2 \lambda^{t+1} e^{I x \Psi} e^{I y \Psi}-2 \lambda^{t} e^{I x \Psi} e^{I y \Psi}+\varnothing \lambda^{t} e^{I(x+1) \Psi} e^{I y \Psi}-\varnothing \lambda^{t} e^{I(x-1) \Psi} e^{I y \Psi}+\emptyset \lambda^{t} e^{I x \Psi} e^{I(y+1) \Psi}-\emptyset \lambda^{t} e^{I x \Psi} e^{I(y-1) \Psi}=$ $2 \mu \lambda^{t} e^{I x \Psi} e^{I(y+1) \Psi}-4 \mu \lambda^{t} e^{I x \Psi} e^{I y \Psi}+2 \mu \lambda^{t} e^{I x \Psi} e^{I(y-1) \Psi}$

Dividing equation (18) throughout by $\lambda^{t} e^{I x \Psi} e^{I y \Psi}$ yields:

$2 \lambda-2+\emptyset e^{I \Psi}-\emptyset e^{-I \Psi}+\emptyset e^{I \Psi}-\emptyset e^{-I \Psi}=2 \mu e^{I \Psi}-4 \mu+2 \mu e^{-I \Psi}$

Making $\lambda$ the subject of the formula in equation (19) gives:

$2 \lambda=(1-2 \mu)+(\mu-\emptyset) e^{I \Psi}+(\mu+\emptyset) e^{-I \Psi}$

By Euler's formula we have, Tinega and Ndede, [20]:

$e^{I \Psi}=\cos \Psi+i \sin \Psi$

$e^{I \Psi}=\cos \Psi-i \sin \Psi$

Substituting equation (21) into equation (20) yields:

$\lambda=(1-2 \mu)+2 \mu \cos \Psi-i 2 \emptyset \sin \Psi$

Separating the real and the imaginary parts and simplifying equation (22) becomes:

$\lambda=(1-2 \mu)+2 \mu \cos \Psi-i 2 \emptyset \sin \Psi$

Considering the real, $\operatorname{Re}(\lambda)$ and the imaginary, $\operatorname{Im}(\lambda)$ parts of $\lambda$ independently, equation (23) becomes:

$\operatorname{Re}(\lambda)=(1-2 \mu)+2 \mu \cos \Psi$

$\operatorname{Im}(\lambda)=-2 \emptyset \sin \Psi$

Where, $\lambda$ is the amplification factor which determines whether the error is bounded in stability. For stable situation, it is required that $|\lambda| \leq 1$.

Taking the maximum value of $\Psi$, that is, $\Psi=90^{\circ}$ for the largest eigenvalue of the amplification factor, equations (24) and (25) become: 
International Journal of Advances in Scientific Research and Engineering (ijasre), Volume 7 (6), 2021

$|R e(\lambda)|=|(1-2 \mu)+2 \mu \cos \Psi|=|(1-2 \mu)|$

Similarly,

$|\operatorname{Im}(\lambda)|=|-2 \emptyset \sin \Psi|=|-2 \emptyset(1)|=|-2 \emptyset|$

From equations (26) and (27) are stable for all values $\mu>0, \emptyset<\frac{1}{2}$. Thus, the FTCS scheme for the energy equation in this research is conditionally stable.

\subsection{Consistency Analysis for the Energy Equation FTCS scheme.}

Putting, $E c \delta \mu=0$, equation (16) the FTCS scheme for the energy equation becomes

$2 \theta_{i, j}^{k+1}-2 \theta_{i, j}^{k}+\emptyset \theta_{i+1, j}^{k}-\emptyset \theta_{i-1, j}^{k}+\emptyset \theta_{i, j+1}^{k}-\emptyset \theta_{i, j-1}^{k}=2 \mu \theta_{i, j+1}^{k}-4 \mu \theta_{i, j}^{k}+2 \mu \theta_{i, j-1}^{k}$

To evaluate the consistency of the energy equation FTCS scheme (28), the Taylor's series expansions as stated by Jain [21] is utilized as shown below:

$$
\begin{aligned}
& \theta_{i, j}^{k+1}=\theta_{i, j}^{k}+(\Delta t) \theta_{t}+\frac{(\Delta t)^{2}}{2} \theta_{t t}+\frac{(\Delta t)^{3}}{6} \theta_{t t t}+\cdots \\
& \theta_{i, j+1}^{k}=\theta_{i, j}^{k}+(\Delta y) \theta_{y}+\frac{(\Delta y)^{2}}{2} \theta_{y y}+\frac{(\Delta y)^{3}}{6} \theta_{y y y}+\cdots \\
& \theta_{i, j-1}^{k}=\theta_{i, j}^{k}-(\Delta y) \theta_{y}+\frac{(\Delta y)^{2}}{2} \theta_{y y}-\frac{(\Delta y)^{3}}{6} \theta_{y y y}+\cdots \\
& \theta_{i+1, j}^{k}=\theta_{i, j}^{k}+(\Delta x) \theta_{x}+\frac{(\Delta x)^{2}}{2} \theta_{x x}+\frac{(\Delta x)^{3}}{6} \theta_{x x x}+\cdots \\
& \theta_{i-1, j}^{k}=\theta_{i, j}^{k}-(\Delta x) \theta_{x}+\frac{(\Delta x)^{2}}{2} \theta_{x x}-\frac{(\Delta x)^{3}}{6} \theta_{x x x}+\cdots
\end{aligned}
$$

Substituting equations (29), (30), (31), (32) and (33) in the implicit scheme (11) we obtain:

$$
\begin{aligned}
& 2\left(\theta_{i, j}^{k}+(\Delta t) \theta_{t}+\frac{(\Delta t)^{2}}{2} \theta_{t t}+\frac{(\Delta t)^{3}}{6} \theta_{t t t}+\cdots\right)-2 \theta_{i, j}^{k}+\emptyset\left(\theta_{i, j}^{k}+(\Delta x) \theta_{x}+\frac{(\Delta x)^{2}}{2} \theta_{x x}+\frac{(\Delta x)^{3}}{6} \theta_{x x x}+\cdots\right)- \\
& \varnothing\left(\theta_{i, j}^{k}-(\Delta x) \theta_{x}+\frac{(\Delta x)^{2}}{2} \theta_{x x}-\frac{(\Delta x)^{3}}{6} \theta_{x x x}+\cdots\right)+\emptyset\left(\theta_{i, j}^{k}+(\Delta y) \theta_{y}+\frac{(\Delta y)^{2}}{2} \theta_{y y}+\frac{(\Delta y)^{3}}{6} \theta_{y y y}+\cdots\right)-\emptyset\left(\theta_{i, j}^{k}-\right. \\
& \left.(\Delta y) \theta_{y}+\frac{(\Delta y)^{2}}{2} \theta_{y y}-\frac{(\Delta y)^{3}}{6} \theta_{y y y}+\cdots\right)=\left(2 \mu \theta_{i, j}^{k}+2 \mu(\Delta y) \theta_{y}+\mu(\Delta y)^{2} \theta_{y y}+\mu \frac{(\Delta y)^{3}}{3} \theta_{y y y}+\cdots\right)-4 \mu \theta_{i, j}^{k}+ \\
& \left(2 \mu \theta_{i, j}^{k}-2 \mu(\Delta y) \theta_{y}+\mu(\Delta y)^{2} \theta_{y y}-\mu \frac{(\Delta y)^{3}}{3} \theta_{y y y}+\cdots\right)
\end{aligned}
$$

Upon simplifying equation (34), we obtain

$2(\Delta t) \theta_{t}+2 \emptyset(\Delta x) \theta_{x}+2 \emptyset(\Delta y) \theta_{y}-2 \mu(\Delta y)^{2} \theta_{y y}+\cdots=0$

Putting $\Delta x=\Delta y=\Delta t=h, \varnothing=\frac{\Delta t}{\Delta x}=\frac{\Delta t}{\Delta y}=1$ and $\mu=\frac{\Delta t}{(\Delta x)^{2}}=\frac{\Delta t}{(\Delta y)^{2}}=\frac{1}{h}$, in equation (34) and dividing the resulting equation by 2 throughout we obtain:

$\theta_{t}+\theta_{x}+\theta_{y}-\theta_{y y}+\cdots=0$

It is noted that equation (36) is the recovered partial differential equation (energy equation). Having recovered the energy equation from the algebraic equation of the FTCS scheme developed, we therefore conclude that the scheme is consistent with the energy equation PDE.

\section{Convergence}

By the Lax-Richtmyer Equivalence Theorem, the achievement of a convergent numerical solution based on a particular finite difference approximation is manifested in the consistency and stability of the finite difference scheme utilized Richtmyer and Morton [22].

\section{Conclusion}


The effect of variable viscosity on the unsteady free convective fluid flow over a vertical stretching sheet in the presence of thermal radiation and viscous dissipation has been discussed. The resulting partial differential equations were discretized using the finite difference method to obtain the FTCS scheme. The results were presented graphically illustrating the details of the heat transfer characteristics on some physical parameters. Further, the stability and consistency analysis of the governing equation has been done using the Von-Neumann analysis and the Taylor's series respectively. The following conclusions may be drawn:

i) A rise in Eckert number leads to enhanced temperature profiles whereas increasing the radiation parameter decreases the temperature profiles.

ii) Increase in variable viscosity parameter implies increased fluid viscosity which in turn leads to an increase in the temperature due to thickening of the thermal boundary layer.

iii) The FTCS scheme is conditionally stable and consistent.

\section{Declaration}

The authors declare that there are no conflicts of interest to disclose.

\section{REFERENCES}

[1] J. Alinejad and S. Samarbakhsh, "Viscous flow over nonlinearly stretching sheet with effects of viscous dissipation", Journal of Applied Mathematics, 2012, Article ID 587834.

[2] A.B. Jafar, S. Shafie and I. Ullah, "Magnetohydrodynamic boundary layer flow of a viscoelastic fluid past a nonlinear stretching sheet in the presence of viscous dissipation effect," Coatings, 2019, 9, 490.

[3] I. Pop and T.Y. Na, "Unsteady flow past a stretching sheet," Mechanics Research Communications, 1996, vol. 23, issue 4, pp.413-422.

[4] R. Nazar, A. Ishak and I. Pop, "Unsteady boundary layer flow over a stretching sheet in a micropolar fluid," International Journal of Physical and Mathematical Sciences, 2008, vol. 2, issue 2, pp.108-112.

[5] E.M.A. Elbashbeshy and D.A. Aldawoody, "Heat transfer over an unsteady stretching surface with variable heat flux in the presence of a heat source or sink," Computers and Mathematics with Applications, 2010, vol. 60, pp. 2806-2811.

[6] J. Phakirappa, P.H. Veena and V.K. Pravin, "Boundary layer flow and heat transfer flow past stretching sheet with temperature gradient dependent heat sink and internal heat generation", International Journal of Modern Engineering Research (IJMER), 2012, vol. 2, issue 5, pp. 3298-3305.

[7] R. Sharma, A. Ishak and I. Pop, "Partial slip flow and heat transfer over a stretching sheet in a nanofluid", Mathematical Problems in Engineering, 2013, http://dx.doi.org/10.1155/2013/724547.

[8] K. Vajravelu, K.V. Prasad and C.O. Ng, "Unsteady convective boundary layer flow of a viscous fluid at a vertical surface with variable fluid properties,” Nonlinear Analysis: Real World Applications, 2013, vol. 14, pp. 55-464.

[9] M.G. Reddy, P. Padma and B. Shankar, "Effects of viscous dissipation and heat source on unsteady MHD flow over a stretching sheet”, Ain Shams Engineering Journal, 2015, vol.6, issue 4, pp. 1195-1201.

[10] V. Malapati and P. Polarapu, "Unsteady MHD free convective heat and mass transfer in a boundary layer flow past a vertical permeable plate with thermal radiation and chemical reaction," Procedia Engineering, 2015, vol. 127, pp. 791-799.

[11] Y.S. Daniel, Y.S. Aziz, Z. Ismail and F. Salah, "Impact of thermal radiation on electrical MHD nanofluid over nonlinear stretching sheet with variable thickness”, Alexandria Engineering Journal, 2017, vol. 57, pp.187-2197.

[12] O.J. Fenuga, A.R. Hassan and P.O. Olanrewaju," Effects of radiation and Eckert number on MHD flow with heat transfer rate near a stagnation point over a non-linear vertical stretching sheet," International Journal of Applied Mechanics and Engineering, 2020, vol. 25, issue 1, pp. 27-36. 
[13] K. Sharma and S. Gupta," Viscous dissipation and thermal radiation effects in MHD flow of Jeffrey nanofluid through impermeable surface with heat generation/absorption," Nonlinear Engineering, 2017, vol. 6, issue 2, pp. 153-166.

[14] G.C. Shit and R. Haldar, "Combined effects of thermal radiation and Hall current on MHD free-convective flow and mass transfer over a stretching sheet with variable viscosity”, Journal of Applied Fluid Mechanics, 2012, vol. 5, issue 2, pp.113-121.

[15] A.M. Salem, "The effects of variable viscosity, viscous dissipation and chemical reaction on heat and mass transfer flow of MHD micropolar fluid along a permeable stretching sheet in a non-Darcian porous medium", Mathematical Problems in Engineering, 2013, Article ID 185074

[16] D. Pal and H. Mondal, "Effects of temperature-dependent viscosity and variable thermal conductivity on MHD- non-Darcy mixed convective diffusion of species over a stretching sheet", Journal of the Egyptian Mathematical Society, 2014, vol. 22, pp.23-133.

[17] S. Shateyi and S.S. Motsa., "Variable viscosity on magnetohydrodynamic flow and heat transfer over an unsteady stretching surface with Hall effect”, Boundary Value Problems, 2010, doi:10.1155/2010/257568.

[18] D. Pal and H. Mondal, "MHD non-Darcian mixed convection heat and mass transfer over a non-linear stretching sheet with Soret Dufour effects and chemical reaction”, Int. Commun. Heat and Mass Transfer, 2011, vol. 38, pp. 463-467.

[19] L. Lapidus and G.F. Pinder, “Numerical Solution of Differential Equations in Science and Engineering”, 1982, John Wiley and Sons Inc.'

[20] A.K. Tinega and C.O. Ndede, "Stability and consistency analysis for central difference scheme for Advection-Diffusion partial differential equation”, International Journal of Science and Research (IJSR), 2016, vol. 8, issue 7, pp. 1046-1049.

[21] M.K. Jain, “Numerical methods for scientific and engineering computation”, Wiley Eastern Ltd, New Delhi, 1984.

[22] R.D. Richtmyer and K.W. Morton, "Difference Methods for Initial Value Problems", $2^{\text {nd }}$ Edition, Interscience Pub., Wiley, New York, 1967

\section{Authors Profile}

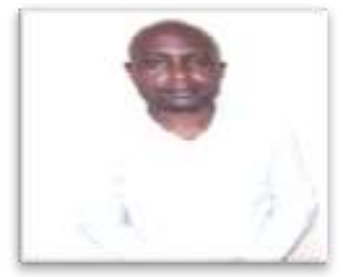

Abraham Osogo Nyakebogo holds a Bachelor of Education Science degree, from Egerton University, Nakuru, Kenya, and a Master of Science degree in Applied Mathematics from Jomo Kenyatta University of Agriculture and Technology (JKUAT), Nairobi, Kenya and is currently pursuing a PhD in applied Mathematics at Kisii University, Kenya. He has much interest in the study of fluid mechanics.

Affiliation: Kisii University, KISII, KENYA.

Email: abrahampsogo@kisiiuniversity.ac.ke

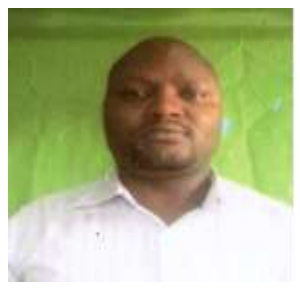

Dr Robert Obogi holds a Bachelor of Education Arts degree from Egerton University, Kenya, Master of Science degree in pure Mathematics from Kenyatta University, and a $\mathrm{PhD}$ in pure mathematics from Jaramogi Oginga Odinga University of Science and Technology (JOOUST), Kenya. He is currently the Dean school of Pure and Applied Sciences (January 2019 to date).

Affiliation: Kisii University, KISII, KENYA.

Email: robogi@kisiiuniversity.ac.ke

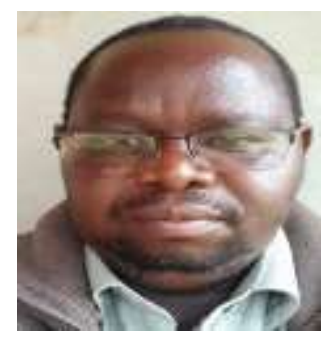

Dr Joash Morara Kerongo holds a Bachelor of Education Arts degree from Egerton University, Kenya, Master of Science degree in Applied Mathematics from Kenyatta University, and a PhD in applied mathematics from Jomo Kenyatta University of Agriculture and Technology (JKUAT), Nairobi, Kenya. He is currently the Ag Registrar Academics at Kisii University.

Affiliation: Kisii University, KISII, KENYA.

Email: jkerongo@kisiiuniversity.ac.ke 